Supplement of

\title{
Variations in export production, lithogenic sediment transport and iron fertilization in the Pacific sector of Drake Passage over the past $400 \mathrm{ka}$
}

María H. Toyos et al.

Correspondence to: María H. Toyos (mtoyos@udec.cl)

The copyright of individual parts of the supplement might differ from the article licence. 
Table S1. Correlation matrix between ${ }^{230}$ Th-normalized MARs of productivity export production proxies and lithogenic indicators for core PS97/093-2.

\begin{tabular}{|c|c|c|c|c|c|c|}
\hline $\begin{array}{l}\text { Litho. MAR } \\
\left.\text { (g/cm }{ }^{2} / \mathrm{kyr}\right)\end{array}$ & $\begin{array}{c}\text { Lithogenic } \\
\text { MAR } \\
\left(\mathrm{g} / \mathrm{cm}^{2} / \mathrm{kyr}\right)\end{array}$ & & & & & \\
\hline $\begin{array}{c}\text { Fe MAR } \\
\left(\mathrm{mg} / \mathrm{cm}^{2} / \mathrm{kyr}\right)\end{array}$ & $\begin{array}{c}r^{2}=0.98 \\
P<0.0001 \\
n=60\end{array}$ & $\begin{array}{c}\text { Fe MAR } \\
\left(\mathrm{mg} / \mathrm{cm}^{2} / \mathrm{kyr}\right)\end{array}$ & & & & \\
\hline $\begin{array}{c}\mathrm{Ba}_{\mathrm{exc}} \text { MAR } \\
\left(\mathrm{mg} / \mathrm{cm}^{2} / \mathrm{kyr}\right)\end{array}$ & $\begin{array}{c}r^{2}=0.44 \\
P<0.0001 \\
n=60\end{array}$ & $\begin{array}{c}r^{2}=0.46 \\
P<0.0001 \\
n=60\end{array}$ & $\begin{array}{c}\mathrm{Ba}_{\mathrm{exc}} \text { MAR } \\
\left(\mathrm{mg} / \mathrm{cm}^{2} / \mathrm{kyr}\right)\end{array}$ & & & \\
\hline $\begin{array}{c}\text { TOC MAR } \\
\left(\mathrm{mg} / \mathrm{cm}^{2} / \mathrm{kyr}\right)\end{array}$ & $\begin{array}{c}r^{2}=0.81 \\
P<0.0001 \\
n=60\end{array}$ & $\begin{array}{c}r^{2}=0.80 \\
P<0.0001 \\
n=60\end{array}$ & $\begin{array}{c}r^{2}=0.48 \\
P<0.0001 \\
n=60\end{array}$ & $\begin{array}{c}\text { TOC MAR } \\
\left(\mathrm{mg} / \mathrm{cm}^{2} / \mathrm{kyr}\right)\end{array}$ & & \\
\hline $\begin{array}{l}\text { Opal MAR } \\
\left(\mathrm{g} / \mathrm{cm}^{2} / \mathrm{kyr}\right)\end{array}$ & $\begin{array}{c}r^{2}=0.89 \\
P<0.0001 \\
n=60\end{array}$ & $\begin{array}{c}r^{2}=0.85 \\
P<0.0001 \\
n=60\end{array}$ & $\begin{array}{c}r^{2}=0.47 \\
P<0.0001 \\
n=60\end{array}$ & $\begin{array}{c}r^{2}=0.57 \\
P<0.0001 \\
n=60\end{array}$ & $\begin{array}{l}\text { Opal MAR } \\
\text { (g/cm²/kyr) }\end{array}$ & \\
\hline $\begin{array}{c}\mathrm{CaCO}_{3} \mathrm{MAR} \\
\left(\mathrm{g} / \mathrm{cm}^{2} / \mathrm{kyr}\right)\end{array}$ & $\begin{array}{c}r^{2}=0.11 \\
P=0.009 \\
n=60\end{array}$ & $\begin{array}{c}r^{2}=0.10 \\
P=0.009 \\
n=60\end{array}$ & $\begin{array}{c}r^{2}=0.03 \\
P=0.128 \\
n=60\end{array}$ & $\begin{array}{c}r^{2}=0.02 \\
P=0.208 \\
n=60\end{array}$ & $\begin{array}{c}r^{2}=0.04 \\
P=0.059 \\
n=60\end{array}$ & $\begin{array}{c}\mathrm{CaCO}_{3} \\
\text { MAR } \\
\left(\mathrm{g} / \mathrm{cm}^{2} / \mathrm{kyr}\right)\end{array}$ \\
\hline
\end{tabular}



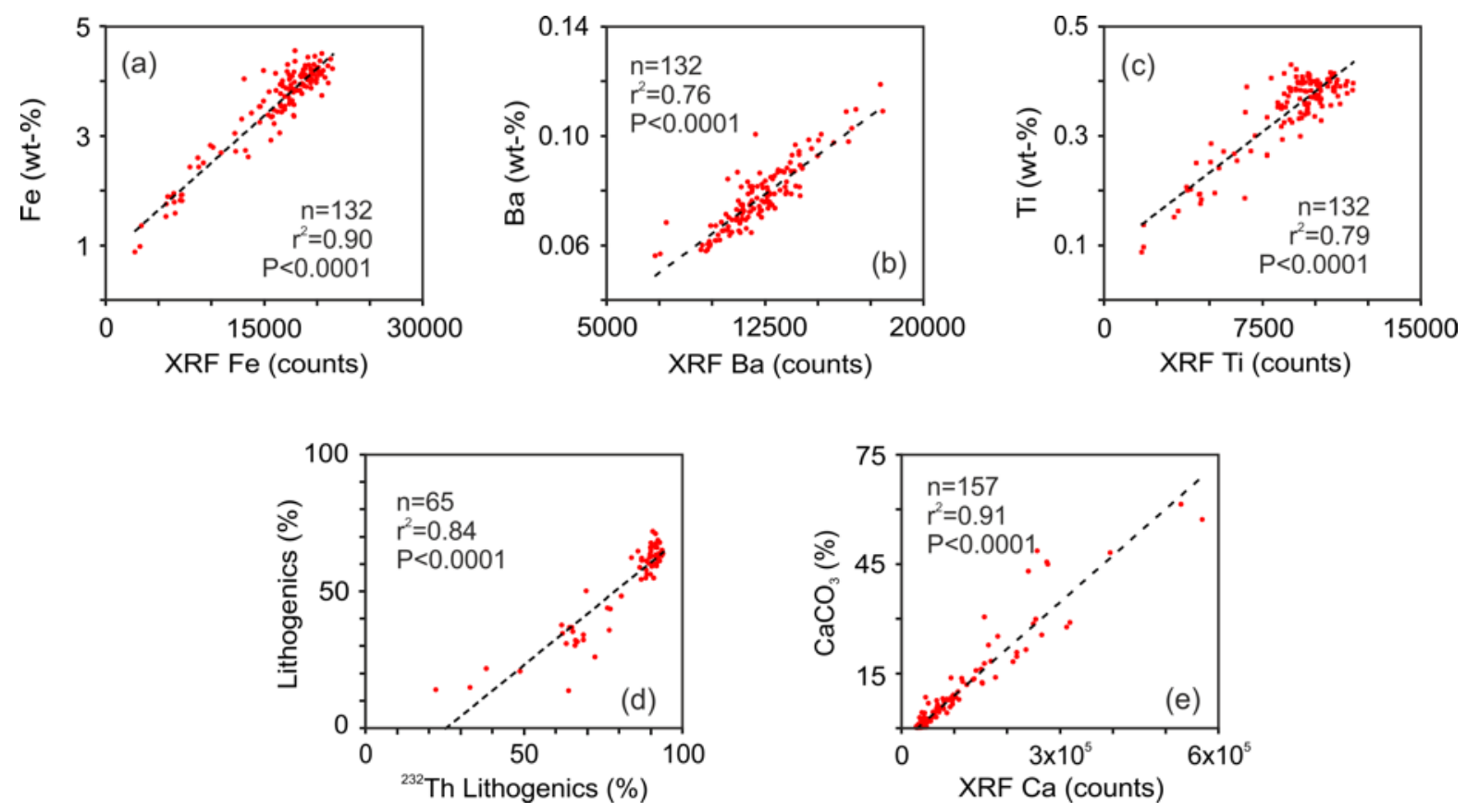

Figure S1. a, b, c: Linear regressions between XRF and measured element concentrations (Fe, Ba, Ti) used to obtain high-resolution element content records. $d$ : Linear regression between lithogenic content obtained by subtraction $\left(\mathrm{Litho} \%=100-\left[\mathrm{CaCO}{ }_{3} \%+\mathrm{opal} \%+\right.\right.$ $(2 \mathrm{xTOC} \%)]$, and lithogenic content obtained by ${ }^{232} \mathrm{Th}$ concentrations. e: Linear regression between $\mathrm{XRF}_{\mathrm{Ca}}$ and measured $\mathrm{CaCO}_{3}$ percentage used to obtain a high-resolution carbonate content record. 

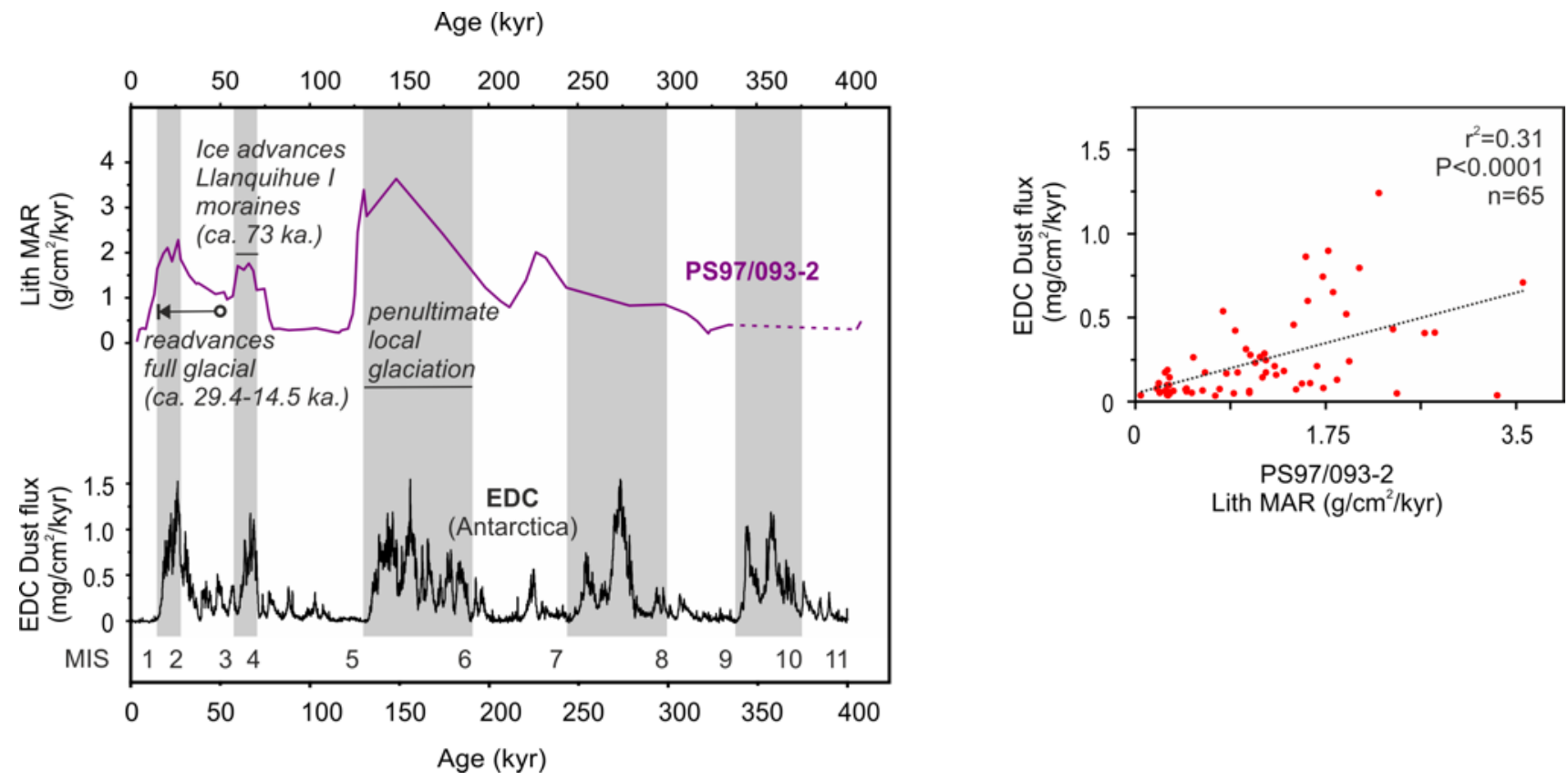

Figure S2. Left: Changes in ${ }^{230}$ Th-normalized Lithogenic MARs of core PS97/093-2 compared to dust fluxes in the EPICA Dome C ice core (Lambert et al., 2008). The inset texts indicate the chronology of the ice advances in Patagonia and Tierra del Fuego according to Rabassa (2008). Vertical grey bars mark glacial stages according to Lisiecki \& Raymo (2005). Marine Isotope Stage (MIS). Right: Linear correlation between PS97/093-2 lithogenic MARs and EDC dust flux.

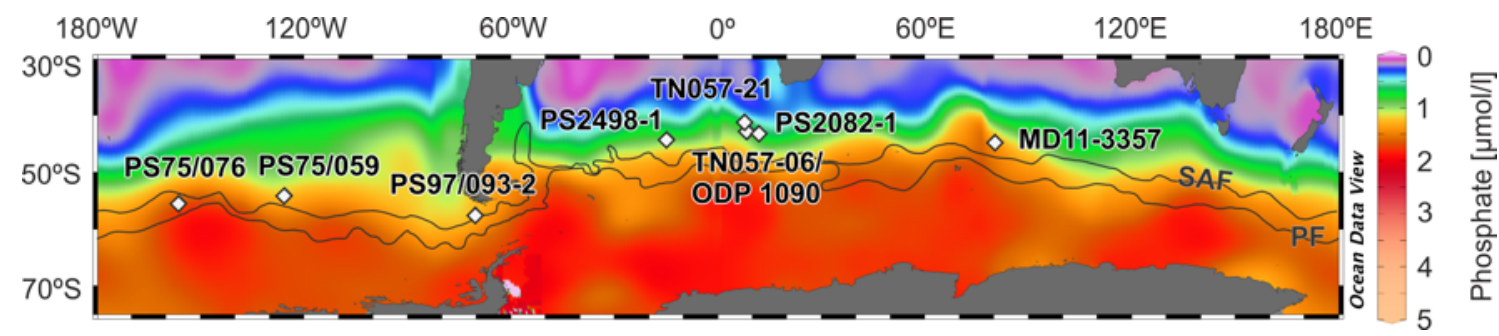

Figure S3. Southern Ocean average surface concentration of phosphate (data from Gouretski \& Koltermann, 2004); Black lines indicate the modern location of the Subantarctic Front (SAF), and Polar Front (PF, Orsi et al., 1995); White diamonds indicate the core locations (PS75/076 and PS75/059, Lamy et al. 2014; PS97/093-2, this study; PS2498-1, TN057-21 and TN057-06, Anderson et al., 2014; ODP1090, Martínez-García et al. 2014; PS2082-1, Nürnberg, 1997 and Frank, 2002; MD11-3357, Thöle et al., 2019). 


\section{References}

Anderson, R. F., Barker, S., Fleisher, M., Gersonde, R., Goldstein, S. L., Kuhn, G., Mortyn, P. G., Pahnke, K. and Sachs, J. P.: Biological response to millennial variability of dust and nutrient supply in the Subantarctic South Atlantic Ocean, Philos. Trans. R. Soc. A Math. Phys. Eng. Sci., 372(2019), doi:10.1098/rsta.2013.0054, 2014.

Frank, M.: Accumulation rate and vertical rain rate of sediment core PS2082-1. PANGAEA, https://doi.org/10.1594/PANGAEA.81101, 2002.

Gouretski, V. V. and Koltermann, K. P.: The World Ocean Circulation Experiment (WOCE) Global Hydrographic Climatology, 35th ed., edited by B. BHS, Hamburg. [online] Available from: http://rda.ucar.edu/datasets/ds285.4/, 2004.

Lambert, F., Delmonte, B., Petit, J. R., Bigler, M., Kaufmann, P. R., Hutterli, M. A., Stocker, T. F., Ruth, U., Steffensen, J. P. and Maggi, V.: Dust - Climate couplings over the past 800,000 years from the EPICA Dome C ice core, Nature, 452(7187), 616-619, doi:10.1038/nature06763, 2008.

Lamy, F., Gersonde, R., Winckler, G., Esper, O., Jaeschke, A., Kuhn, G., Ullermann, J., Martinez-Garcia, A., Lambert, F. and Kilian, R.: Increased Dust Deposition in the Pacific Southern Ocean During Glacial Periods, Science., 343(6169), 403407, doi:10.1126/science.1245424, 2014.

Lisiecki, L. E. and Raymo, M. E.: A Pliocene-Pleistocene stack of 57 globally distributed benthic $\delta 18$ O records, Paleoceanography, 20(1), doi:10.1029/2004PA001071, 2005.

Martínez-Garcia, A., Sigman, D. M., Ren, H., Anderson, R. F., Straub, M., Hodell, D. A., Jaccard, S. L., Eglinton, T. I. and Haug, G. H.: Iron Fertilization of the Subantarctic Ocean During the Last Ice Age, Science., 343(6177), 1347-1350, doi:10.1126/science.1246848, 2014.

Nürnberg, C. C., Bohrmann, G., Schlüter, M. and Frank, M.: Barium accumulation in the Atlantic sector of the Southern Ocean: Results From 190,000-year records, Paleoceanography, 12(4), 594-603, doi:10.1029/97PA01130, 1997.

Rabassa, J.: Late Cenozoic Glaciations in Patagonia and Tierra del Fuego, Dev. Quat. Sci., 11, 151-204, doi:10.1016/S15710866(07)10008-7, 2008.

Thöle, L. M., Amsler, H. E., Moretti, S., Auderset, A., Gilgannon, J., Lippold, J., Vogel, H., Crosta, X., Mazaud, A., Michel, E., Martínez-García, A. and Jaccard, S. L.: Glacial-interglacial dust and export production records from the Southern Indian Ocean, Earth Planet. Sci. Lett., 525, doi:10.1016/j.eps1.2019.115716, 2019. 